\title{
Coding Schemes for Implementation of Fault Tolerant Parrallel Filter
}

\author{
Nutenki Siddhartha, G Renuka \\ Department of ECE, SR Engineering College, Hasanparthy, Warangal, Telangana, India.
}

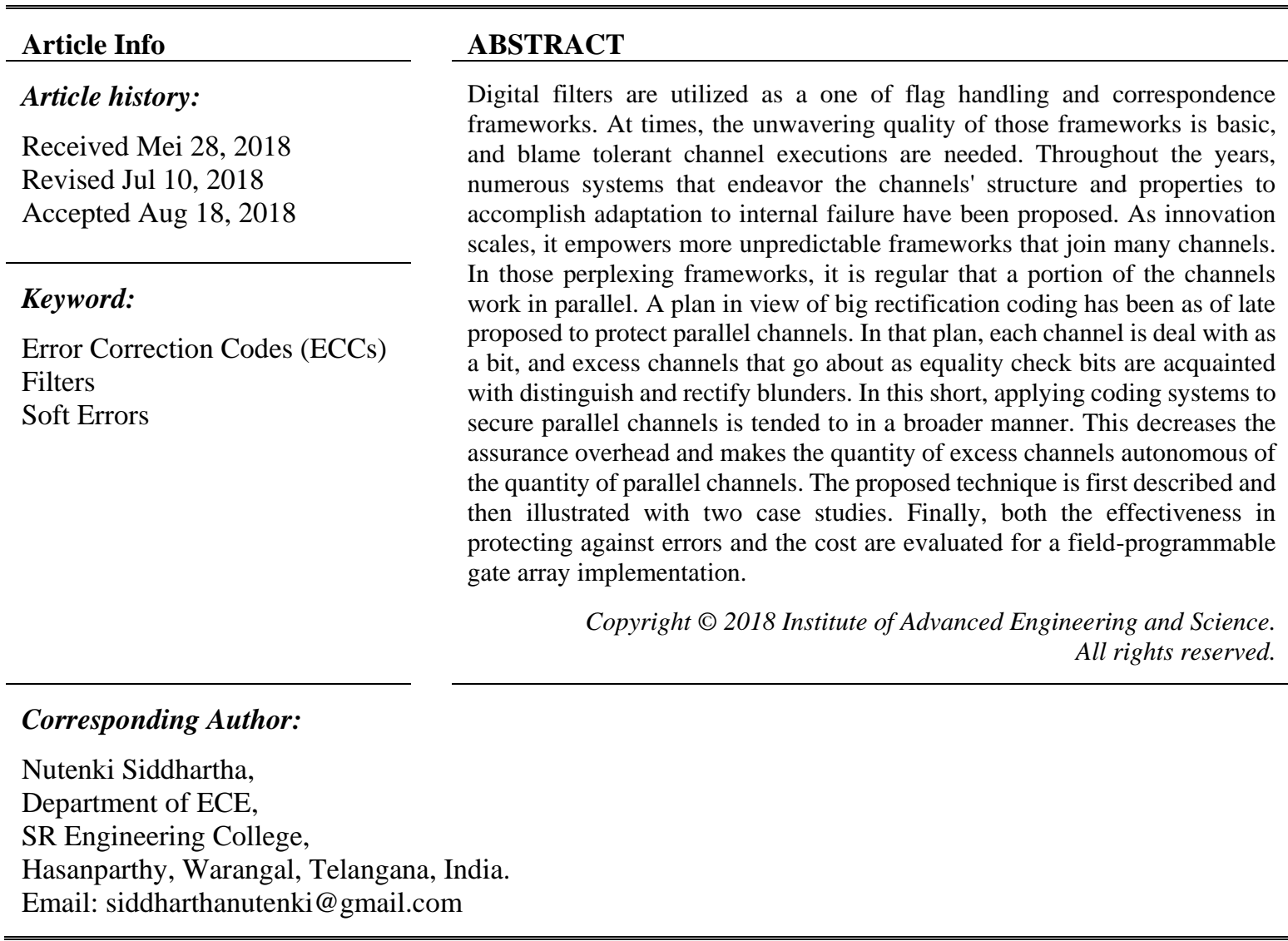

\section{INTRODUCTION}

Electronic circuits are progressively present in and space applications where dependability is basic. In those applications, the circuits need to give some level of adaptation to non-critical failure. This need is additionally expanded by the inherent unwavering quality difficulties of cutting edge CMOS innovations that incorporate, e.g., producing varieties and delicate blunders. Various strategies can be utilized to shield a circuit from blunders. Those range from adjustments in the assembling procedure of the circuits to decrease the quantity of mistakes to including repetition at the rationale or framework level to guarantee that blunders don't influence the framework usefulness.

Channels are regularly utilized as a part of electronic frameworks to underline motions in certain recurrence ranges and reject motions in other recurrence ranges. In circuit hypothesis, a channel is an electrical system that adjusts the abundancy as well as stage qualities of a flag regarding recurrence. In a perfect world, a channel won't add new frequencies to the info flag, nor will it change the segment frequencies of that flag, yet it will change the relative amplitudes of the different recurrence segments as well as their stage connections. Today channels are generally utilized as a part of number of uses which in view of car, restorative, and space where unwavering quality of segments in computerized electronic circuits is basic. Channels or the like are basic in the operation of most electronic circuits. There are various bases of characterizing channels and these cover in a wide range of ways; there is no straightforward progressive grouping. As the behavioral properties 
of flag changes the strategies of separating it will be contrast. Being particular with channel, the computerized channels have huge applications in advanced flag preparing. Sifting is likewise a class of flag preparing, the characterizing highlight of channels being the total or incomplete concealment of some part of the flag. It is in this manner in light of a legitimate concern for anybody associated with electronic circuit configuration to be able to create channel circuits fit for meeting a given arrangement of particulars. In flag handling, an advanced channel is a gadget or process that expels some undesirable segment or highlight from a flag. Advanced channels are utilized for two general purposes; division of signs that have been joined, and reclamation of signs that have been contorted somehow. Frequently, this implies expelling a few frequencies and not others with a specific end goal to smother meddling signs and decrease foundation commotion.

Parallel Processing and correspondence frameworks. As a rule, Parallel channels are normally found in current flag the channels play out an indistinguishable preparing on various approaching signs from there is an inclination to utilize different input-multiple yield frameworks.

The security of computerized channels has been broadly contemplated. For instance, blame tolerant executions in light of the utilization of deposit number frameworks or number-crunching codes have been proposed. The utilization of decreased accuracy replication or word-level insurance has been likewise examined another alternative to perform blunder remedy is to utilize two distinctive channel usage in parallel. Every one of those systems concentrate on the security of a solitary channel.

This concise examinations the assurance of parallel channels utilizing more broad coding strategies. Specifically, a key contrast with ECCs is that both channel sources of info and yields are numbers. Hence, not just a zero or a one can be utilized for the coding (as finished with ECCs). This can be abused, as appeared in whatever is left of this brief, to give mistake remedy by including just two repetitive channels paying little heed to the quantity of parallel channels. The decreased number of repetitive channels does not influence the capacity of the plan to revise mistakes however diminishes the usage cost. In whatever is left of this short, to begin with, the parallel channels and the current ECC-based security plot are depicted. At that point, the proposed coding plan is given and delineated a couple of useful contextual analyses. At last, the contextual analyses are assessed for a field-programmable door cluster (FPGA) usage and contrasted and the already proposed ECC-based system.

\subsection{Concept of Fault Tolerance}

Various procedures can be utilized to shield a circuit from mistakes. Those range from changes in the assembling procedure of the circuits to lessen the quantity of blunders to including repetition at the rationale or framework level to guarantee that mistakes don't influence the framework usefulness. Computerized Filters are a standout amongst the most generally utilized flag handling circuits and a few procedures have been proposed to shield them from blunders. There are number of techniques used to recognize flaws and the activities important to adjust the issues inside circuit. Advanced channels are broadly utilized as a part of flag handling and correspondence frameworks. There are distinctive adaptation to internal failure ways to deal with customary computational circuits and the DSP circuits. Now and again, the unwavering quality of those frameworks is basic, and blame tolerant channel executions are required. Throughout the years, numerous methods that endeavor the channels structure and properties to accomplish adaptation

\section{LITERATURE REVIEW}

[1] In this paper, adaptation to non-critical failure construct framework based with respect to Error Correction Codes (ECCs) utilizing VHDL is outlined, executed, and tried. It recommends that with the assistance of ECCs i.e. Blunder Correction Codes there will be more ensured Parallel channel circuit has been conceivable. The channel they have utilized for blunder location and revision are for the most part limited motivation reaction (FIR) channels. They have been utilized Hamming Codes for blame adjustment in which they takes a square of $\mathrm{k}$ bits and produces a piece of $\mathrm{n}$ bits by including $\mathrm{n}-\mathrm{k}$ equality check bits. The equality check bits are XOR mixes of the k information bits. By appropriately planning those mixes it is conceivable to distinguish and revise blunders. In this plan they have utilized repetitive module in which the information and equality check bits are store $d$ and can be recuperated later regardless of the possibility that there is a blunder in one of the bits. This is finished by re - registering the equality check bits and contrasting the outcomes and the qualities put away. Along these lines utilizing hamming codes mistake can be recognized and revised inside the circuit.

[2] In this paper, Triple Modular Redundancy (TMR) and Hamming Codes have been utilized to ensure distinctive circuits against Single Event Upsets (SEUs). In this paper, the utilization of a Novel Hamming approach on FIR Filters is considered and executed with a specific end goal to give low unpredictability, lessen deferral and territory proficient assurance methods for higher bits information. A novel Hamming code is proposed in this paper, to build the proficiency of higher information bits. In this paper, they 
have proposed method used to illustrate, how the part of overhead because of mixing the repetition bits, their consequent evacuation, cushion to cushion postpone in the decoder and utilization of aggregate range of FIR channel for higher bits are decreased. These depend on the novel hamming code usage in the FIR channel rather than regular hamming code used to ensure FIR channel. In this plan Hamming code utilized for transmission of 7-bit information thing.

[3] In this paper, the outline of a FIR channel with self checking capacities in view of the deposit checking is broke down. Typically the arrangement of deposits used to check the consistency of the aftereffects of the FIR channel are based of theoretic contemplations about the dynamic range accessible with a picked set of buildups, the math qualities of the blunders caused by a blame and on the normal for the channel execution. This examination is frequently hard to perform and to acquire adequate blame scope the arrangement of picked buildups is overestimated. Gotten result and consequently requires that Instead, in this paper they have demonstrated how utilizing a comprehensive blame infusion battles permits to proficiently choose the best arrangement of buildups. Test comes about originating from blame infusion crusades on a 16 taps FIR channel showed that by watching the happened mistakes and the identification modules relating to various deposit has been conceivable to decrease the quantity of discovery module, while paying a little lessening of the level of SEUs that can be distinguished. Paired rationale overwhelms the equipment execution of DSP frameworks

[4] In this paper they have proposed engineering for the execution of blame - tolerant calculation inside a high throughput multirate equalizer for a deviated remote LAN. The zone overhead is limited by abusing the logarithmic structure of the Modulus Replication Residue Number System (MRRNS). They had exhibited that for our framework the zone cost to amend a blame in a solitary computational channel is $82.7 \%$. Adaptation to non-critical failure inside MRRNS design is executed through the expansion of excess channels. This paper has displayed a point by point investigation of the cost of executing single blame adjustment ability in a FIR channel utilizing the MRRNS. The blame tolerant design makes utilization of the mathematical properties of the MRRNS, and has been appeared to give critical range funds when contrasted and general systems. This engineering likewise requires couple of extra parts to be outlined, as indistinguishable excess channels are utilized, and the polynomial mapping stages are just extended from the first segments.

\section{Main Objectives of proposed method:}

There are various objectives over the protection of digital signal processing circuits. The main objectives are given as follows-

1). To achieve fault free digital circuit.

2). To detect and correct errors in digital circuit with more accuracy.

3). To reduce the overhead, needed for protection from error.

4). To improve efficiency.

5). To develop the application area from lower to higher order application.

\section{ECC-BASED PROTECTION OF PARALLEL FILTERS}

The impulse response $h[n]$ completely defines a discrete time filter that performs the following operation on the incoming signal $x[n]$ :

$$
y[n]=\sum_{l=0}^{\infty} x[n-l] \cdot h[l] .
$$

This property can be exploited in the case of parallel filters that operate on different incoming signals, as shown on Figure. 1. In this case, four filters with the same response process the incoming signals $x 1[n], x$ $2[n], x 3[n]$, and $x 4[n]$ to produce four outputs $y 1[n], y 2[n], y 3[n]$, and $y 4[n]$.

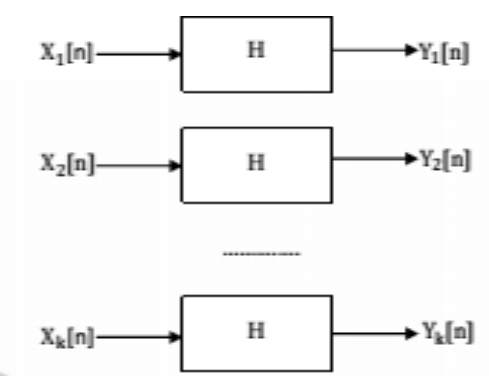

Figure 1. Block diagram for Parallel FIR Filters 
To detect and correct errors, each filter can be viewed as a bit in an ECC, and redundant filters can be added to form parity check bits. This is also illustrated in Figure. 2, where three redundant filters are used to form the parity check bits of a classical single error correction Hamming code [14]. Those correspond to the outputs $z 1[n], z 2[n]$, and $z 3[n]$. Errors can be detected by checking if

$$
\begin{aligned}
& z_{1}[n]=y_{1}[n]+y_{2}[n]+y_{3}[n] \\
& z_{2}[n]=y_{1}[n]+y_{2}[n]+y_{4}[n] \\
& z_{3}[n]=y_{1}[n]+y_{3}[n]+y_{4}[n]
\end{aligned}
$$

When some of those checks fail, an error is detected. The error can be corrected based on which specifi checks failed. For example, an error on filter $y 1$ will cause errors on the checks of $z 1, z 2$, and $z 3$

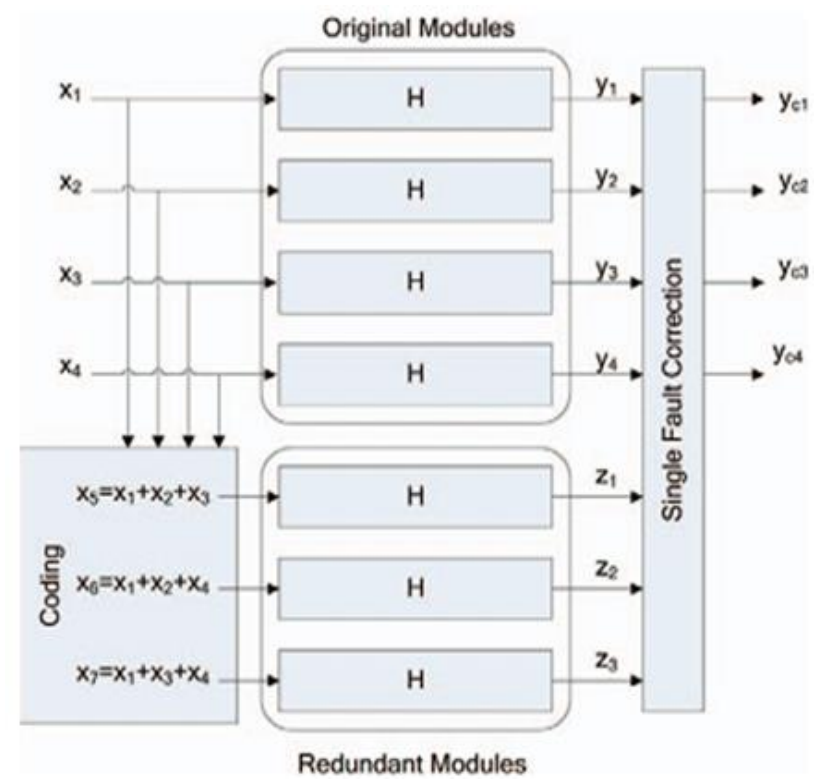

Figure 2. ECC-based scheme for four filters and a Hamming code.

\section{CODING FOR FAULT-TOLERANT PARALLEL FILTERS}

The proposed scheme is illustrated in Figure. 3 for the case of four parallel filters. The input signals are encoded using a matrix with arbitrary coefficients to make the signals that enter the four original and two redundant filters. In its more general form, this coding matrix $A$ can be formulated as

$$
A=\left(\begin{array}{cccc}
a_{11} & a_{12} & a_{13} & a_{14} \\
a_{21} & a_{22} & a_{23} & a_{24} \\
a_{31} & a_{32} & a_{33} & a_{34} \\
a_{41} & a_{42} & a_{43} & a_{44} \\
a_{51} & a_{52} & a_{53} & a_{54} \\
a_{61} & a_{62} & a_{63} & a_{64}
\end{array}\right)
$$




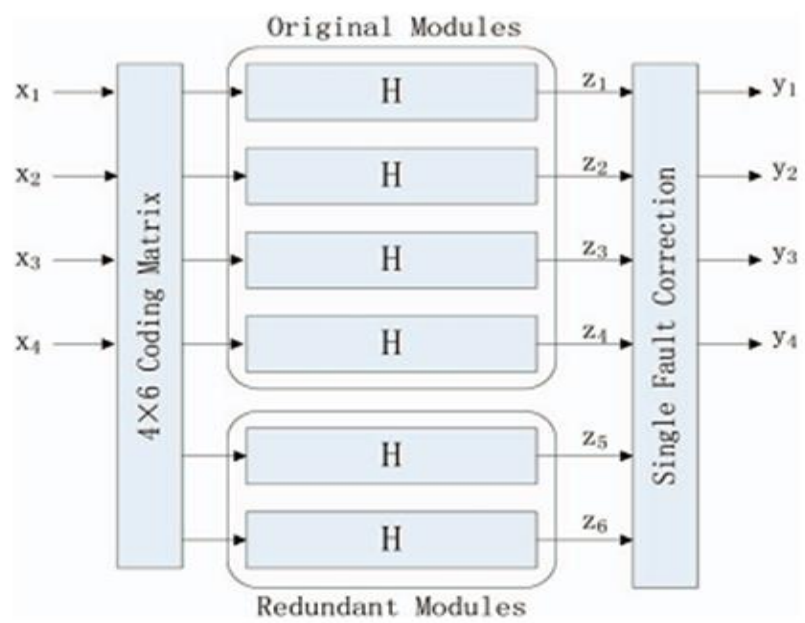

Figure 3. Proposed coding scheme in general form

With this coding scheme, the outputs of the filters, i.e., y1[n], y2[n], y3[n], and y4[n], can be obtained as follows:

$$
\left(\begin{array}{l}
y_{1} \\
y_{2} \\
y_{3} \\
y_{4}
\end{array}\right)_{1235}=\left(A_{1235}\right)^{-1}\left(\begin{array}{l}
z_{1} \\
z_{2} \\
z_{3} \\
z_{5}
\end{array}\right)
$$

where $A 1235$ is a sub matrix of $A$, including the first, second, third, and fifth rows. This process can be repeated with different submatrixes of $A$, for example, with $A 1236, A 2345$, and $A 2346$. In the error-free case, all the recovered versions of $y 1[n], y 2[n], y 3[n]$, and $y 4[n]$ will be the same. When there are differences, an error is detected. For xample, suppose that

$$
\left(\begin{array}{l}
y_{1} \\
y_{2} \\
y_{3} \\
y_{4}
\end{array}\right)_{1235} \neq\left(\begin{array}{l}
y_{1} \\
y_{2} \\
y_{3} \\
y_{4}
\end{array}\right)_{1236}\left(\begin{array}{l}
y_{1} \\
y_{2} \\
y_{3} \\
y_{4}
\end{array}\right)_{2345}=\left(\begin{array}{l}
y_{1} \\
y_{2} \\
y_{3} \\
y_{4}
\end{array}\right)_{2346}
$$

Which means that there is an error among filters $\left\{\begin{array}{lllll}1 & 2 & 3 & 5 & 6\end{array}\right\}$ and that filters $\left\{\begin{array}{llllll}2 & 3 & 4 & 5 & 6\end{array}\right\}$ are correct. Therefore, the faulty filter is filter 1 . Then, the error can be corrected by taking the final outputs from a set that does not include filter 1 .

The error correction and detection logic can be simplified assuming that there is only a single error. In that case, checking only that, for each recovered set, the sums of the values $y 1[n]+y 2[n]+y 3[n]+y 4[n]$ are equal is enough. In more detail, four checks are needed, each involving five filters and excluding one. For example, if branch 1 is excluded, the error checking would be

$$
\left\{\begin{array}{l}
s_{1}^{1}=\bar{w}_{2345}\left(z_{2} z_{3} z_{4} z_{5}\right)^{T} \\
s_{1}^{2}=\bar{w}_{2346}\left(z_{2} z_{3} z_{4} z_{6}\right)^{T} \\
e_{1}=s_{1}^{1}-s_{1}^{2}
\end{array}\right.
$$

in which $w^{-} 2345=[1111](A 2345)-1$, and $w^{-} 2346=[1111](A 2346)-1$. 


\section{RESULTS}

The written Verilog HDL Modules have successfully simulated and verified using Modelsim III 6.4b and synthesized using Xilinx ISE 13.2.

Simulation Result
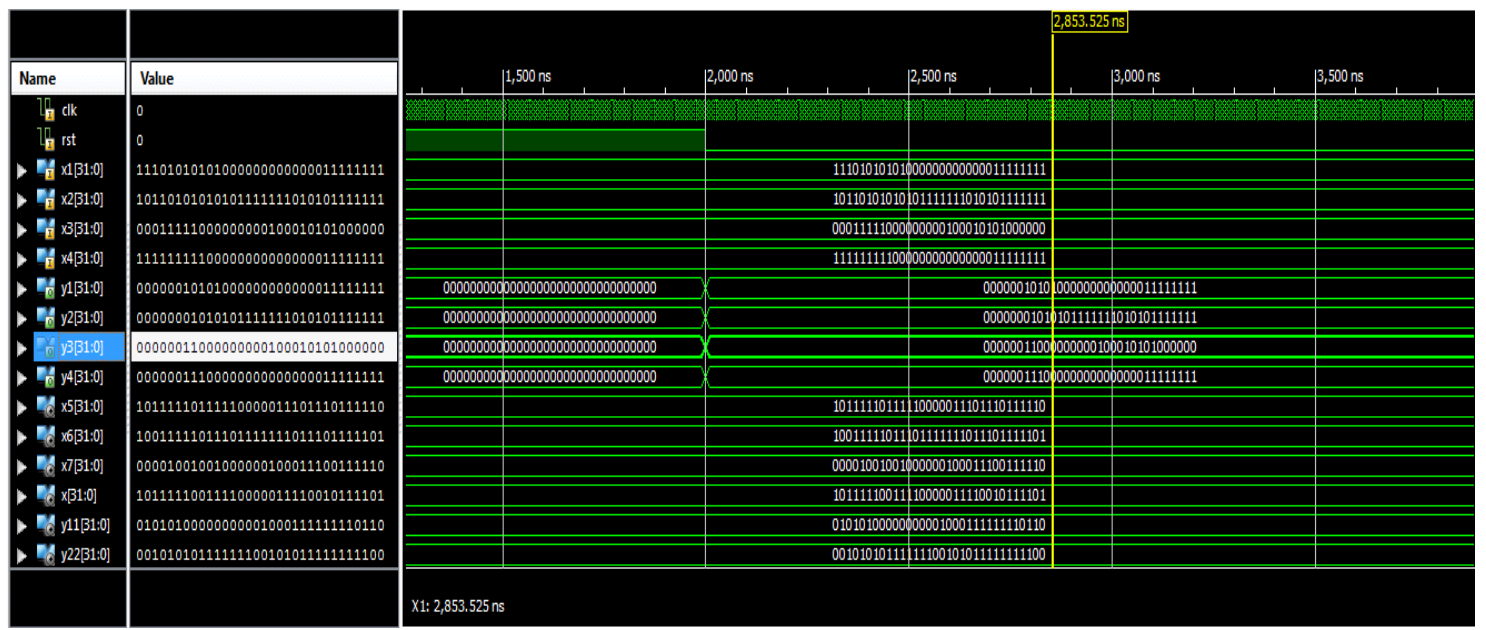

Figure 4. Simulation Result

In Figure 5 we observed that simulation results and Figure. 6 and Figure.7 shows that RTL schematic and Technology schematic respectively.

Table 1. Design Summary

\begin{tabular}{cccc}
\hline \multicolumn{4}{c}{ Device Utilization Summary (Estimated Values) } \\
\hline Logic Utilization & Used & Available & Utilization \\
Number of Slices & 225 & 4656 & $4 \%$ \\
Number of Slice & 229 & 9312 & $2 \%$ \\
$\quad$ FlipFLOPS & 421 & 9312 & $4 \%$ \\
$\begin{array}{c}\text { Number of 4 input } \\
\text { LUTs }\end{array}$ & 234 & 232 & $100 \%$ \\
$\begin{array}{c}\text { Number of Bonded } \\
\text { IOBs }\end{array}$ & 1 & 24 & $4 \%$ \\
Number of GCLKs & 1 & & \\
\hline
\end{tabular}

\section{RTL Schematic}

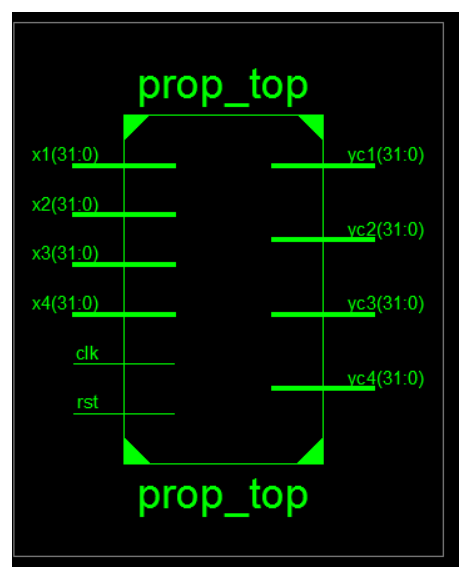

Figure 5. RTL Schematic 


\section{Technology Schematic}

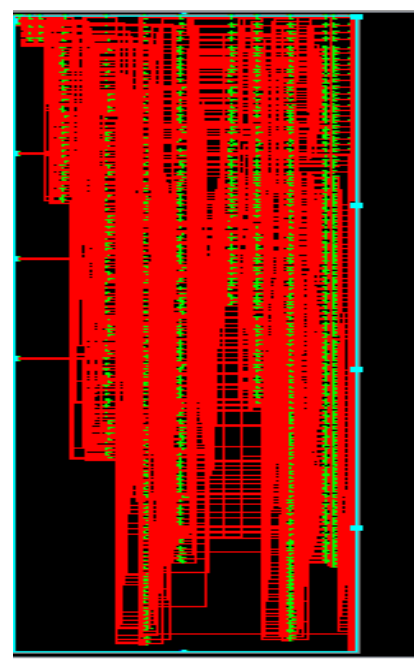

Figure 6. Technology Schematic

\section{Timing Report}

\begin{tabular}{|c|c|c|c|c|}
\hline Cell: in->out & fanout & $\begin{array}{l}\text { Gate } \\
\text { Delay }\end{array}$ & $\begin{array}{r}\text { Net } \\
\text { Delay }\end{array}$ & Logical Name (Net Name) \\
\hline 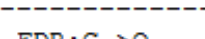 & & -----1 & ----- & 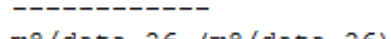 \\
\hline FDR : C->Q & 1 & 0.514 & 0.357 & m8/data_26 (m8/data_26) \\
\hline OBUF : $I->0$ & & 3.169 & & yc1_25_OBUF $\quad($ yc1<25>) \\
\hline 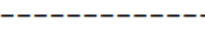 & 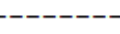 & --------1 & ------ & \\
\hline Total & & $4.040 \mathrm{~ns}$ & $\begin{array}{l}(3.683 \\
191.28\end{array}$ & $\begin{array}{l}\text { hs logic, } 0.357 \mathrm{~ns} \text { route) } \\
\text { logic, } 8.8 \% \text { route) }\end{array}$ \\
\hline
\end{tabular}

Figure 7. Timing Report

\section{CONCLUSION}

In this paper a new scheme to protect parallel filters that are commonly found in modern signal processing circuits has presented. The approach is based on applying ECCs to the parallel filters outputs to detect and correct errors. The technique can be used for parallel filters that have the same response and process different input signals. An objectives has also been discussed to show the effectiveness of the scheme in terms of error correction and problem definition also shows the overheads. The proposed scheme can also make system price lower. Proposed work will result in more efficient fault tolerant system using parallel IIR filters based on ECCs, which will meet the goal to achieve low power consumption, increase area of application and high speed.

\section{REFERENCES}

[1] P. P. Vaidyanathan, Multirate Systems and Filter Banks, Englewood Cliffs, N.J., USA: Prentice Hall, 1993.

[2] A. Sibille, C. Oestges and A. Zanella, MIMO: From Theory to Implementation, New York, NY, USA: Academic, 2010.

[3] N. Kanekawa, E. H. Ibe, T. Suga and Y. Uematsu, Dependability in Electronic Systems: Mitigation of Hardware Failures, Soft Errors, and ElectroMagnetic Disturbances, New York, NY, USA: Springer Verlag, 2010.

[4] M. Nicolaidis, "Design for soft error mitigation," IEEE Trans. Device Mater. Rel., vol. 5, no. 3, pp. 405-418, Sep. 2005.

[5] C. L. Chen and M. Y. Hsiao, "Error-correcting codes for semiconductor memory applications: A state-of-the-art review,” IBM J. Res. Develop.vol. 28, no. 2, pp. 124-134, Mar. 1984.

[6] A. Reddy and P. Banarjee "Algorithm-based fault detection for signal processing applications," IEEE Trans. Comput., vol. 39, no. 10, pp. 1304-1308, Oct. 1990.

[7] T. Hitana and A. K. Deb, "Bridging concurrent and non-concurrent error detection in FIR filters, " in Proc. Norchip Conf., 2004, pp. 75-78. 
[8] Y.-H. Huang, "High-efficiency soft-error-tolerant digital signal processing using fine -grain subword-detection Processing," IEEE Trans. Very Large Scale Integr. (VLSI) Syst., vol. 18, no. 2, pp. 291-304, Feb. 2010.

[9] S. Pontarelli, G. C. Cardarilli, M. Re, and A. Salsano, "Totally fault tolerant RNS based FIR filters," in Proc. IEEE IOLTS, Jul. 2008, pp. 192-194.

\section{BIOGRAPHIES OF AUTHORS}

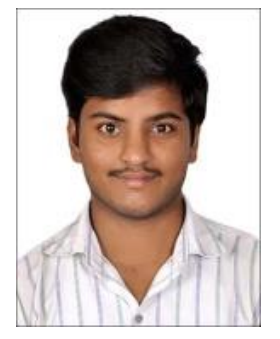

Nutenki Siddhartha is pursuing Master of Technology in Electronic Design Technology in SR Engineering College, Warangal, and Telangana. He has completed his B.Tech from Kamala Institute of Technology and Science, Karimnagar, Telangana in 2014. His areas of interests are VLSI, Digital Signal Processing and Computer Networks. He Published 1 Poster in ICRTEECT-2017.

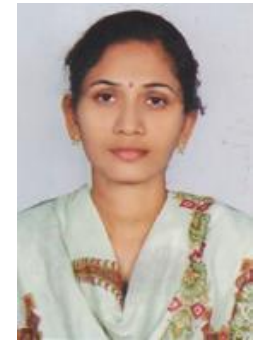

G. Renuka working as assistant professor of Electronics and Communication Engineering, S R Engineering College, Warangal. She has 12 years of teaching experience. She has obtained B.Tech (ECE) Degree from Ramappa Engineering College Warangal, Andhra Pradesh, India in 2003 and M.Tech (Digital Communications) Degree from Kakatiya University, Andhra Pradesh, India in 2009. Pursuing her Ph.D under JNTUH. She is a member of IETE, and her research areas include VLSI, Communications. 